İsmail Aslan*

\title{
Traveling Waves of DDEs with Rational Nonlinearity
}

DOI 10.1515/ijnsns-2016-0028

Received February 16, 2016; accepted June 6, 2016

\begin{abstract}
It has been found that the dynamical behavior of many complex physical systems can be properly described by nonlinear DDEs. However, in the related literature, research focusing on such equations with rational nonlinearity is rare. Hence, the present study makes an attempt to fill the existing gap. To this end, we consider two distinct DDEs with rational nonlinearity. We observed that the model equations assume three kinds of traveling wave solutions; hyperbolic, trigonometric and rational including kink-type solitary waves and singular periodic solutions. Our discussion is based on the auxiliary equation method.
\end{abstract}

Keywords: Traveling waves, solitons, differential-difference equation, auxiliary equation method

PACS $^{\circledR}$ (2010). 05.45.Yv, 04.20.Jb, 02.30.Jr

\section{Introduction}

Nonlinearity is everywhere around us. Consequently, nonlinear sciences have emerged in a variety of disciplines such as physical, biological, chemical, economical, social and engineering, and in particular, nonlinear differential-difference equations (DDEs) or lattice equations. The fundamental challenges of nonlinear science are those of description and prediction. Based on the observation of a certain phenomena, we wish to know how to describe what we see now and how to determine the later behavior. Nonlinear DDEs are important models for scientists and engineers in attacking problems involving the description and prediction of the behavior of complex physical systems. Nonlinear DDEs are considered to be hybrid systems because the spatial variable $n$ is discrete while the time $t$ is usually kept continuous. As a result of intense and ingenious research, many integrable nonlinear DDEs have been developed since the original work of Fermi, Pasta and

*Corresponding author: İsmail Aslan, Department of Mathematics, İzmir Institute of Technology, Urla, İzmir 35430, Turkey,

E-mail: ismailaslan@iyte.edu.tr
Ulam [1]. For instance, the Volterra lattice equation, the Toda lattice equation, the Ablowitz-Ladik lattice equation, the discrete sine-Gordon equation, the discrete (modified) KdV equation, the Suris lattice and so forth [2]. These are just a few examples enough to exhibit the variety and complexity of lattice equations. The mentioned equations are mostly in the form $\dot{u}_{n}=$ $P\left(u_{n-1}, u_{n}, u_{n+1}\right)$ where $P$ is a polynomial function of its arguments and the dependent variable $u_{n}$ is assumed to be a function $u(n, t)$ of a lattice variable $n \in \mathbb{Z}$. Although many particular equations of this general class have appeared in the literature, there are very few systematic accounts available for nonlinear DDEs with rational nonlinearity $[3,4]$ :

$$
\dot{u}_{n}=R\left(u_{n-1}, u_{n}, u_{n+1}\right),
$$

where $R$ is a rational function of its arguments, $n \in \mathbb{Z}$, $u_{n}(t)=u(n, t)$ is the displacement of the $n$th particle from the equilibrium position. This fact forces us to devote this study to the following DDEs with rational nonlinearity $[5,6]$ :

$$
\begin{gathered}
\dot{u}_{n}=\frac{u_{n-1}-u_{n+1}}{1-u_{n-1}+u_{n+1}}, \\
\dot{u}_{n}=\frac{2\left(u_{n-1}-u_{n+1}\right) u_{n}^{2}\left(1-u_{n}^{2}\right)}{\left(u_{n-1}+u_{n}\right)\left(u_{n}+u_{n+1}\right)} .
\end{gathered}
$$

Equation (2) is related to Volterra equation. The aim of this work is to obtain exact solutions of eqs (2) and (3) which may be of physical interest and complement the existing literature. The existence of a special class of explicit solutions called traveling waves is one of the most fundamental questions regarding nonlinear DDEs. A soliton, which was first discovered in 1834 by Russell [7], is a special traveling wave that after a collision with another soliton eventually emerges unscathed. Solitary solutions of DDEs have caught much attention due to the fact that discrete spacetime may be the most radical and logical viewpoint of reality [8].

The rest of this study is organized as follows. In Section 2, we first describe the algorithm nonlinear DDEs. Subsequently, in Sections 3 and 4, we apply the method to the eqs (2) and (3) to search for exact traveling wave solutions. Finally, some concluding remarks are given in Section 5. 


\section{The auxiliary equation method for DDEs}

Let us consider a system of $M$ nonlinear DDEs in the form [9]

$$
\begin{aligned}
& \Omega\left(\mathbf{u}_{\mathrm{n}+\mathrm{p}_{1}}(\mathbf{x}), \ldots, \mathbf{u}_{\mathrm{n}+\mathrm{p}_{k}}(\mathbf{x}), \ldots, \mathbf{u}_{\mathrm{n}+\mathrm{p}_{1}}^{\prime}(\mathbf{x}), \ldots,\right. \\
& \left.\mathbf{u}_{\mathrm{n}+\mathrm{p}_{k}}^{\prime}(\mathbf{x}), \ldots, \mathbf{u}_{\mathrm{n}+\mathrm{p}_{1}}^{(r)}(\mathbf{x}), \ldots, \mathbf{u}_{\mathrm{n}+\mathrm{p}_{k}}^{(r)}(\mathbf{x})\right)=0,
\end{aligned}
$$

where the dependent variable $\mathbf{u}_{\mathrm{n}}$ have $M$ components $u_{i, \mathbf{n}}$ and so do its shifts; the continuous variable $\mathbf{x}$ has $N$ components $x_{i}$; the discrete variable $\mathbf{n}$ has $Q$ components $n_{j}$; the $k$ shift vectors $\mathbf{p}_{i} \in Z^{Q}$; and $\mathbf{u}^{(r)}(\mathbf{x})$ denotes the collection of mixed derivative terms of order $r$. We search exact solutions of eq. (4) by means of the wave transformation

$$
\begin{gathered}
\mathbf{u}_{\mathrm{n}+\mathrm{p}_{\mathrm{s}}}(\mathbf{x})=\mathbf{U}_{\mathbf{n}+\mathbf{p}_{s}}\left(\xi_{\mathbf{n}}\right), \xi_{\mathbf{n}}=\sum_{i=1}^{Q} d_{i} n_{i}+\sum_{j=1}^{N} c_{j} x_{j}+\zeta, \\
(s=1,2, \ldots, k)
\end{gathered}
$$

where the coefficients $c_{1}, c_{2}, \ldots, c_{N}, d_{1}, d_{2}, \ldots, d_{Q}$ and the phase $\zeta$ are all constants. Then, eq. (4) reduces to a system of DDEs

$$
\begin{aligned}
& \Omega\left(\mathbf{U}_{\mathbf{n}+p_{1}}\left(\xi_{\mathbf{n}}\right), \ldots, \mathbf{U}_{\mathbf{n}+\mathbf{p}_{k}}\left(\xi_{\mathbf{n}}\right), \ldots, \mathbf{U}_{\mathbf{n}+\mathbf{p}_{1}}^{\prime}\left(\xi_{\mathbf{n}}\right), \ldots,\right. \\
& \left.\mathbf{U}_{\mathbf{n}+\mathbf{p}_{k}^{\prime}}^{\prime}\left(\xi_{\mathbf{n}}\right), \ldots, \mathbf{U}_{\mathbf{n}+\mathbf{p}_{1}}^{(r)}\left(\xi_{\mathbf{n}}\right), \ldots, \mathbf{U}_{\mathbf{n}+\mathbf{p}_{k}}^{(r)}\left(\xi_{\mathbf{n}}\right)\right)=0 .
\end{aligned}
$$

According to the simplest equation method, we expand the solutions $\mathbf{U}_{\mathbf{n}}\left(\xi_{\mathbf{n}}\right)$ of eq. (6) in a finite series form

$$
\mathbf{U}_{\mathbf{n}}\left(\xi_{\mathbf{n}}\right)=\sum_{l=0}^{m} A_{l}\left(\frac{\psi^{\prime}\left(\xi_{\mathbf{n}}\right)}{\psi\left(\xi_{\mathbf{n}}\right)}\right)^{l}, A_{l}=\text { const., } A_{m} \neq 0,
$$

where $m$ is a positive integer which is determined by balancing the highest order nonlinear term(s) and the highest-order derivative term(s) in eq. (6) and the function $\psi\left(\xi_{\mathbf{n}}\right)$ is a solution of the auxiliary equation

$$
\psi^{\prime \prime}\left(\xi_{\mathbf{n}}\right)+\mu \psi\left(\xi_{\mathbf{n}}\right)=0
$$

where $\mu$ is an arbitrary parameter and prime denotes derivative with respect to $\xi_{n}$. Then, we have the shift formula

$$
\frac{\psi^{\prime}\left(\xi_{\mathbf{n}_{ \pm} \mathbf{p}_{s}}\right)}{\psi\left(\xi_{\mathbf{n} \pm \mathbf{p}_{s}}\right)}=\frac{\frac{\psi^{\prime}\left(\xi_{\mathbf{n}}\right)}{\psi\left(\xi_{\mathbf{n}}\right)} \pm \varepsilon \frac{\sqrt{\delta-4 \varepsilon \mu}}{2} f\left(\frac{\sqrt{\delta-4 \varepsilon \mu}}{2} \varphi_{s}\right)}{1 \pm \frac{2}{\sqrt{\delta-4 \varepsilon \mu}} \frac{\psi^{\prime}\left(\xi_{\mathbf{n}}\right)}{\psi\left(\xi_{\mathbf{n}}\right)} f\left(\frac{\sqrt{\delta-4 \varepsilon \mu}}{2} \varphi_{s}\right)},
$$

where $\varepsilon \in\{0, \pm 1\}, \delta \in\{0,4\}, \varphi_{s}=p_{s 1} d_{1}+p_{s 2} d_{2}+\cdots+$ $p_{s Q} d_{Q}, p_{s j}$ is the $j$ th component of the shift vector $\mathbf{p}_{s}$, and

$$
f\left(\frac{\sqrt{\delta-4 \varepsilon \mu}}{2} \varphi_{s}\right)=\left\{\begin{array}{l}
\tanh \left(\sqrt{-\mu} \varphi_{s}\right), \text { if } \varepsilon=1, \delta=0, \mu<0 \\
\tan \left(\sqrt{\mu} \varphi_{s}\right), \text { if } \varepsilon=-1, \delta=0, \mu>0 \\
\varphi_{s}, \text { if } \varepsilon=0, \delta=4, \mu=0
\end{array}\right.
$$

In addition, we have the uniform shift function

$$
\mathbf{U}_{\mathbf{n} \pm \mathbf{p}_{s}}\left(\xi_{\mathbf{n}}\right)=\sum_{l=0}^{m} A_{l}\left(\frac{\frac{\psi^{\prime}\left(\xi_{\mathbf{n}}\right)}{\psi\left(\xi_{\mathbf{n}}\right)} \pm \varepsilon \frac{\sqrt{\delta-4 \varepsilon \mu}}{2} f\left(\frac{\sqrt{\delta-4 \varepsilon \mu}}{2} \varphi_{s}\right)}{1 \pm \frac{2}{\sqrt{\delta-4 \varepsilon \mu}} \frac{\psi^{\prime}\left(\xi_{\mathbf{n}}\right.}{\psi\left(\xi_{\mathbf{n}}\right)} f\left(\frac{\sqrt{\delta-4 \varepsilon \mu}}{2} \varphi_{s}\right)}\right)^{l}, A_{m} \neq 0 .
$$

Moreover, by means of eq. (7), the degree of $\mathbf{U}_{\mathbf{n}}\left(\xi_{\mathbf{n}}\right)$ is defined as $D\left[\mathbf{U}_{\mathbf{n}}\left(\xi_{\mathbf{n}}\right)\right]=m$ from which the degree of other expressions, such as

$$
\begin{aligned}
D\left[\mathbf{U}_{\mathbf{n}}^{(r)}\left(\xi_{\mathbf{n}}\right)\right]= & m+r, D\left[\left(\mathbf{U}_{\mathbf{n}}^{(r)}\left(\xi_{\mathbf{n}}\right)\right)^{\beta}\right]=\beta(m+r), \\
& D\left[\left(\mathbf{U}_{\mathbf{n}}\left(\xi_{\mathbf{n}}\right)\right)^{\alpha}\left(\mathbf{U}_{\mathbf{n}}^{(r)}\left(\xi_{\mathbf{n}}\right)\right)^{\beta}\right]=\alpha m+\beta(m+r),
\end{aligned}
$$

can be obtained. Then, balancing the highest-order derivative term and the highest order nonlinear term(s) in $\mathbf{U}_{\mathbf{n}}\left(\xi_{\mathbf{n}}\right)$ as in the continuous case, the degree $m$ of eqs (7) and (11) can be easily determined from eq. (6). The leading terms of $\mathbf{U}_{\mathbf{n} \pm \mathbf{p}_{\mathbf{s}}}\left(\mathbf{p}_{s} \neq 0\right)$ will not have any effect on the balancing procedure since the term $\mathbf{U}_{\mathbf{n} \pm \mathbf{p}_{s}}$ can be considered as being of degree zero in $\frac{\psi^{\prime}\left(\xi_{n}\right)}{\psi\left(\xi_{n}\right)}$. Inserting eqs (7) and (11) together with eq. (8) into eq. (6), equating the coefficients of $\left(\frac{\psi^{\prime}\left(\xi_{\mathrm{n}}\right)}{\psi\left(\xi_{\mathrm{n}}\right)}\right)^{l}(l=0,1,2, \ldots)$ to zero, we obtain a system of nonlinear algebraic equations from which the unspecified constants $A_{l}, d_{i}, c_{j}$ and $\mu$ can be explicitly determined. Finally, substituting these results into (7) will lead to different kinds of exact traveling wave solutions for eq. (4).

\section{Traveling waves of eq. (2)}

By means of the wave transformation

$$
u_{n}=U_{n}\left(\xi_{n}\right), \xi_{n}=d n+k t+\chi,
$$

where $d$ and $k$ are real parameters to be specified, while $\chi$ denotes the phase shift, eq. (2) changes to the equation

$$
k U_{n}^{\prime}\left(1-U_{n-1}+U_{n+1}\right)-\left(U_{n-1}-U_{n+1}\right)=0,
$$

where prime denotes ordinary derivative with respect to the new independent variable $\xi_{n}$. Then, we assume that eq. (13) has a solution in the form 


$$
U_{n}=A_{0}+A_{1}\left(\frac{\psi^{\prime}}{\psi}\right), A_{1} \neq 0
$$

where $\psi=\psi\left(\xi_{n}\right)$ is a solution of eq. (8), while $A_{0}$ and $A_{1}$ remains arbitrary to be determined later on. Now, the solutions can be discussed in three cases as follows.

\subsection{Hyperbolic solutions}

In case $\mu<0$, depending upon eq. (11), we get the shift formula

$$
U_{n \pm 1}=A_{0}+A_{1}\left(\frac{\frac{\psi^{\prime}}{\psi} \pm \sqrt{-\mu} \tanh (d \sqrt{-\mu})}{1 \pm \frac{1}{\sqrt{-\mu}}\left(\frac{\psi^{\prime}}{\psi}\right) \tanh (d \sqrt{-\mu})}\right) .
$$

Then, substituting eqs (14) and (15) combined with eq. (8) into eq. (13), clearing the denominator, setting the coefficients of $\left(\frac{\psi^{\prime}}{\psi}\right)^{l}(l=0,2,4)$ to zero, we arrive at a system of nonlinear algebraic equations for $A_{0}, A_{1}, d, k$ and $\mu$. It is observed that a solution of the resulting system is

$$
A_{0}=A_{0}, A_{1}=-\frac{\tanh (d \sqrt{-\mu})}{2 \sqrt{-\mu}}, k=-\frac{\sinh (2 d \sqrt{-\mu})}{\sqrt{-\mu}},
$$

which gives raise a hyperbolic function solution to eq. (2) in the form

$$
\begin{aligned}
u_{n}(t)= & A_{0}-\frac{1}{2} \tanh (d \sqrt{-\mu}) \\
& \left(\frac{C_{1} \sinh \left(\sqrt{-\mu} \xi_{n}\right)+C_{2} \cosh \left(\sqrt{-\mu} \xi_{n}\right)}{C_{1} \cosh \left(\sqrt{-\mu} \xi_{n}\right)+C_{2} \sinh \left(\sqrt{-\mu} \xi_{n}\right)}\right),
\end{aligned}
$$

where $\xi_{n}=d n-\frac{\sinh (2 d \sqrt{-\mu})}{\sqrt{-\mu}} t+\chi$, while $A_{0}, d, \chi, \mu(<0), C_{1}$ and $C_{2}$ remain arbitrary.

\subsection{Trigonometric solutions}

In case $\mu>0$, based on eq. (11), the following shift formula:

$$
U_{n \pm 1}=A_{0}+A_{1}\left(\frac{\frac{\psi^{\prime}}{\psi} \mp \sqrt{\mu} \tan (d \sqrt{\mu})}{1 \pm \frac{1}{\sqrt{\mu}}\left(\frac{\psi^{\prime}}{\psi}\right) \tan (d \sqrt{\mu})}\right)
$$

is obtained. Then substituting eqs (14) and (18) coupled with eq. (8) into eq. (13), clearing the denominator, setting the coefficients of $\left(\frac{\psi^{\prime}}{\psi}\right)^{l}(l=0,2,4)$ to zero, one obtains a system of nonlinear algebraic equations for $A_{0}, A_{1}, d, k$ and $\mu$. A solution of the resulting system is

$$
A_{0}=A_{0}, A_{1}=-\frac{\tan (d \sqrt{\mu})}{2 \sqrt{\mu}}, k=-\frac{\sin (2 d \sqrt{\mu})}{\sqrt{\mu}},
$$

which provides a trigonometric function solution to eq. (2) in the form

$u_{n}(t)=A_{0}-\frac{1}{2} \tan (d \sqrt{\mu})\left(\frac{-C_{1} \sin \left(\sqrt{\mu} \xi_{n}\right)+C_{2} \cos \left(\sqrt{\mu} \xi_{n}\right)}{C_{1} \cos \left(\sqrt{\mu} \xi_{n}\right)+C_{2} \sin \left(\sqrt{\mu} \xi_{n}\right)}\right)$,

where $\xi_{n}=d n-\frac{\sin (2 d \sqrt{\mu})}{\sqrt{\mu}} t+\chi$, while $A_{0}, d, \chi, \mu(>0), C_{1}$ and $C_{2}$ remain arbitrary.

\subsection{Rational solutions}

In case $\mu=0$, in view of eq. (11), the following shift formula

$$
U_{n \pm 1}=A_{0}+A_{1}\left(\frac{\frac{\psi^{\prime}}{\psi}}{1 \pm\left(\frac{\psi^{\prime}}{\psi}\right) d}\right)
$$

is obtained. Then substituting eqs (14) and (21) along with eq. (8) into eq. (13), clearing the denominator, setting the coefficients of $\left(\frac{\psi^{\prime}}{\psi}\right)^{l}(l=2,4)$ to zero, results in a system of nonlinear algebraic equations for $A_{0}, A_{1}$, dand $k$. A solution set of the resulting system is found to be

$$
A_{0}=A_{0}, A_{1}=d, k=-2 d,
$$

which yields a rational function solution to eq. (2) in the form

$$
u_{n}(t)=A_{0}+d\left(\frac{C_{1}}{C_{1}+C_{2}(d n-2 d t+\chi)}\right),
$$

where $A_{0}, d, \chi, C_{1}$ and $C_{2}$ remain arbitrary.

\section{Traveling waves of eq. (3)}

As before, the wave transformation

$$
u_{n}=U_{n}\left(\xi_{n}\right), \xi_{n}=d n+k t+\chi,
$$

where $d$ and $k$ are real parameters to be specified, while $\chi$ denotes the phase shift, takes eq. (3) into the form

$k U_{n}^{\prime}\left(U_{n-1}+U_{n}\right)\left(U_{n}+U_{n+1}\right)-2\left(U_{n-1}-U_{n+1}\right) U_{n}^{2}\left(1-U_{n}^{2}\right)=0$,

where prime denotes ordinary derivative with respect to the new independent variable $\xi_{n}$. Following the same procedure, we search for special solutions of eq. (25) in the form 


$$
U_{n}=A_{0}+A_{1}\left(\frac{\psi^{\prime}}{\psi}\right), A_{1} \neq 0,
$$

where $\psi=\psi\left(\xi_{n}\right)$ is a solution of eq. (8), while $A_{0}$ and $A_{1}$ are arbitrary constants to be found. From now on, some details will be omitted for the sake of brevity since the procedure is similar to that of Section 3.

\subsection{Hyperbolic solutions}

In case $\mu<0$, substituting eq. (26) and $U_{n \pm 1}$ along with eq. (8) into eq. (25), clearing the denominator, setting the coefficients of $\left(\frac{\psi^{\prime}}{\psi}\right)^{l}(l=0,1, \ldots, 6)$ to zero, we derive a system of nonlinear algebraic equations for $A_{0}, A_{1}, d, k$ and $\mu$. From the obtained algebraic system, we get the solutions

$$
\begin{gathered}
A_{0}=-\frac{1}{2} \tanh (d \sqrt{-\mu}), A_{1}= \pm \frac{\tanh (d \sqrt{-\mu})}{2 \sqrt{-\mu}}, \\
k=-\frac{\tanh (d \sqrt{-\mu})}{\sqrt{-\mu}} ; \\
A_{0}=\frac{1}{2} \tanh (d \sqrt{-\mu}), A_{1}= \pm \frac{\tanh (d \sqrt{-\mu})}{2 \sqrt{-\mu}}, \\
k=-\frac{\tanh (d \sqrt{-\mu})}{\sqrt{-\mu}} .
\end{gathered}
$$

Here and then, we order the signs will be in a vertical manner. Setting the parameter values eqs (27) and (28) into the expression (26), some solutions of eq. (3) in terms of hyperbolic functions can be constructed like

$$
\begin{aligned}
u_{n}(t)= & -\frac{1}{2} \tanh (d \sqrt{-\mu}) \pm \frac{1}{2} \tanh (d \sqrt{-\mu}) \\
& \left(\frac{C_{1} \sinh \left(\sqrt{-\mu} \xi_{n}\right)+C_{2} \cosh \left(\sqrt{-\mu} \xi_{n}\right)}{C_{1} \cosh \left(\sqrt{-\mu} \xi_{n}\right)+C_{2} \sinh \left(\sqrt{-\mu} \xi_{n}\right)}\right), \\
u_{n}(t)= & \frac{1}{2} \tanh (d \sqrt{-\mu}) \pm \frac{1}{2} \tanh (d \sqrt{-\mu}) \\
& \left(\frac{C_{1} \sinh \left(\sqrt{-\mu} \xi_{n}\right)+C_{2} \cosh \left(\sqrt{-\mu} \xi_{n}\right)}{C_{1} \cosh \left(\sqrt{-\mu} \xi_{n}\right)+C_{2} \sinh \left(\sqrt{-\mu} \xi_{n}\right)}\right),
\end{aligned}
$$

where $\xi_{n}=d n-\frac{\tanh (d \sqrt{-\mu})}{\sqrt{-\mu}} t+\chi$, while $d, \chi, \mu(<0), C_{1}$ and $C_{2}$ remain arbitrary.

\subsection{Trigonometric solutions}

In case $\mu>0$, substituting eq. (26) and $U_{n \pm 1}$ together with eq. (8) into eq. (25), clearing the denominator, setting the coefficients of $\left(\frac{\psi^{\prime}}{\psi}\right)^{l}(l=0,1, \ldots, 6)$ to zero, we derive a system of nonlinear algebraic equations for $A_{0}, A_{1}, d, k$ and $\mu$. Solving the resulting system, we get the solution sets

$A_{0}=\mp \frac{1}{2} i \tan (d \sqrt{\mu}), A_{1}=-\frac{\tan (d \sqrt{\mu})}{2 \sqrt{\mu}}, k=-\frac{\tan (d \sqrt{\mu})}{\sqrt{\mu}}$,

$A_{0}=\mp \frac{1}{2} i \tan (d \sqrt{\mu}), A_{1}=\frac{\tan (d \sqrt{\mu})}{2 \sqrt{\mu}}, k=-\frac{\tan (d \sqrt{\mu})}{\sqrt{\mu}}$.

Setting the parameter values eqs (31) and (32) into the expression (26), some solutions of eq. (3) in terms of trigonometric functions can be constructed such as

$$
\begin{aligned}
u_{n}(t)= & \mp \frac{1}{2} i \tan (d \sqrt{\mu}) \\
& -\frac{1}{2} \tan (d \sqrt{\mu})\left(\frac{-C_{1} \sin \left(\sqrt{\mu} \xi_{n}\right)+C_{2} \cos \left(\sqrt{\mu} \xi_{n}\right)}{C_{1} \cos \left(\sqrt{\mu} \xi_{n}\right)+C_{2} \sin \left(\sqrt{\mu} \xi_{n}\right)}\right),
\end{aligned}
$$

$$
\begin{aligned}
u_{n}(t)= & \mp \frac{1}{2} i \tan (d \sqrt{\mu}) \\
& +\frac{1}{2} \tan (d \sqrt{\mu})\left(\frac{-C_{1} \sin \left(\sqrt{\mu} \xi_{n}\right)+C_{2} \cos \left(\sqrt{\mu} \xi_{n}\right)}{C_{1} \cos \left(\sqrt{\mu} \xi_{n}\right)+C_{2} \sin \left(\sqrt{\mu} \xi_{n}\right)}\right),
\end{aligned}
$$

where $\xi_{n}=d n-\frac{\tan (d \sqrt{\mu})}{\sqrt{\mu}} t+\chi$, while $d, \chi, \mu(>0), C_{1}$ and $C_{2}$ remain arbitrary.

\subsection{Rational solutions}

In case $\mu=0$, substituting eq. (26) and $U_{n \pm 1}$ together with eq. (8) into eq. (25), clearing the denominator, setting the coefficients of $\left(\frac{\psi^{\prime}}{\psi}\right)^{l}(l=2,3, \ldots, 6)$ to zero, we derive a system of nonlinear algebraic equations for $A_{0}, A_{1}, d$ and $k$. The resulting system gives the solution

$$
A_{0}=0, A_{1}= \pm \frac{d}{2}, k=-2 d,
$$

Inserting the parameter values eq. (35) into eq. (26), we arrive at a rational function solution of eq. (3) such as

$$
u_{n}(t)= \pm \frac{d}{2}\left(\frac{C_{1}}{C_{1}+C_{2}(d n-2 d t+\chi)}\right),
$$

where $d, \chi, C_{1}$ and $C_{2}$ remain arbitrary.

Remark 1. We observe a kink-type solitary wave solution and a singular traveling wave solution if we set " $\mu=-1$, 
$C_{1} \neq 0$ and $C_{2}=0$ " or " $\mu=-1, C_{1}=0$ and $C_{2} \neq 0$ ", respectively, in the solution (17), which are

$$
\begin{aligned}
& u_{n}(t)=A_{0}-\frac{1}{2} \tanh (d) \tanh (d n-\sinh (2 d) t+\chi), \\
& u_{n}(t)=A_{0}-\frac{1}{2} \tanh (d) \operatorname{coth}(d n-\sinh (2 d) t+\chi),
\end{aligned}
$$

where $A_{0}, d$ and $\chi$ remain arbitrary (see Figures 1 and 2).

Similarly, by setting " $\mu=1, C_{1} \neq 0$ and $C_{2}=0$ " or " $\mu=1, C_{1}=0$ and $C_{2} \neq 0$ ", respectively, one can extract the following formal periodic wave solutions from the solution (20)

$$
\begin{aligned}
& u_{n}(t)=A_{0}+\frac{1}{2} \tan (d) \tan (d n-\sin (2 d) t+\chi), \\
& u_{n}(t)=A_{0}-\frac{1}{2} \tan (d) \cot (d n-\sin (2 d) t+\chi),
\end{aligned}
$$

where $A_{0}, d$ and $\chi$ remain arbitrary.

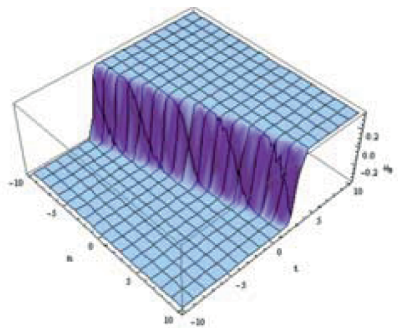

(a)

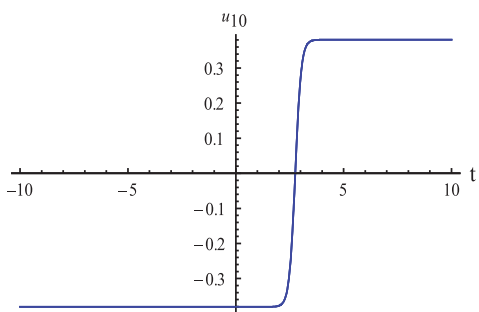

(d)

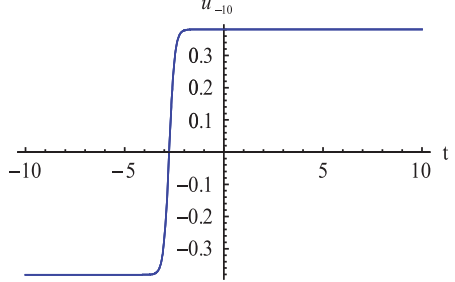

(b)

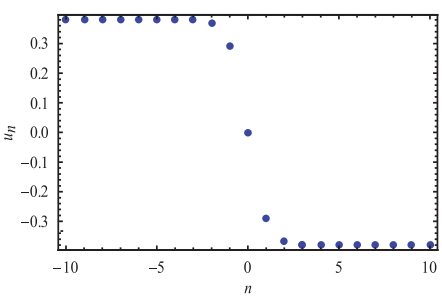

(e)

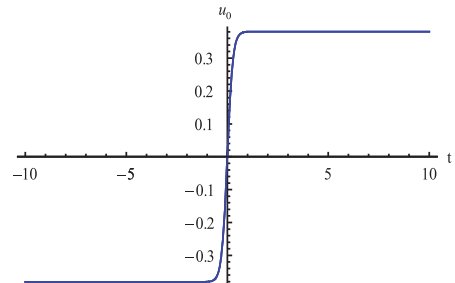

(c)

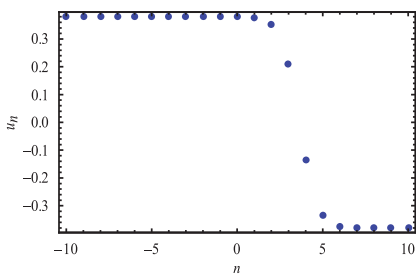

(f)

Figure 1: A profile of the kink-type solitary wave solution (37) with $A_{0}=\chi=0$ and $d=1$ : (a) $-10 \leq t \leq 10, n=0, \pm 1, \pm 2, \ldots, \pm 10$; (b) $-10 \leq t \leq 10, n=-10$; (c) $-10 \leq t \leq 10, n=0$; (d) $-10 \leq t \leq 10, n=10$; (e) $t=0, n=0, \pm 1, \pm 2, \ldots, \pm 10$; (f) $t=1, n=0, \pm 1, \pm 2, \ldots, \pm 10$.

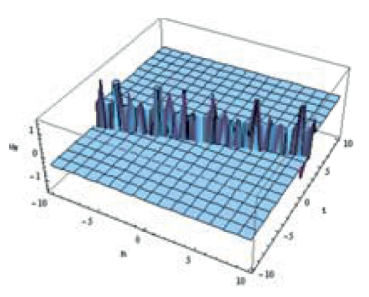

(a)

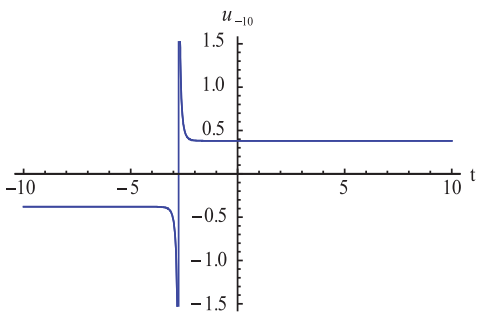

(b)

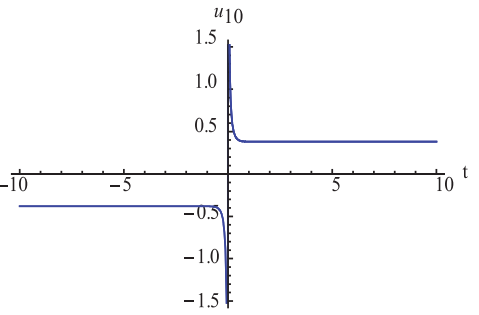

(c)

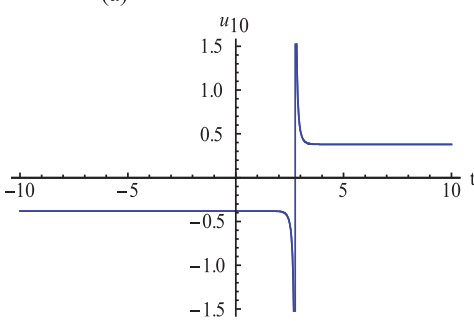

(d)

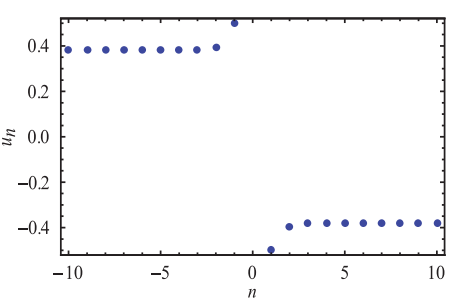

(e)

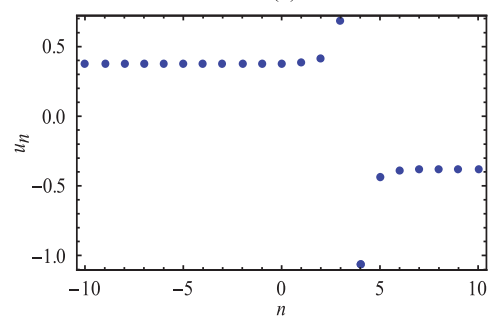

(f)

Figure 2: A profile of the singular traveling wave solution (38) with $A_{0}=\chi=0$ and $d=1:(\mathrm{a})-10 \leq t \leq 10, n=0, \pm 1, \pm 2, \ldots, \pm 10$; (b) $-10 \leq t \leq 10, n=-10$; (c) $-10 \leq t \leq 10, n=0$; (d) $-10 \leq t \leq 10, n=10$; (e) $t=0, n=0, \pm 1, \pm 2, \ldots, \pm 10$; (f) $t=1, n=0, \pm 1, \pm 2, \ldots, \pm 10$. 
Remark 2. Obviously, like in Section 3, setting special values to the arbitrary parameters in eqs (29) and (30) leads to kink-type solitary wave and singular traveling wave solutions.

\section{Conclusion}

While traveling waves is most fully developed for PDEs, these solutions also occur in a number of other types of equations such as nonlinear DDEs. The applications of DDEs permeate all branches of physical sciences. A great deal of study has been devoted to such equations in the related literature. In this study, we primarily focused on DDEs with rational nonlinearity because of their rare treatment. Using one of the existing powerful methods, namely, the auxiliary equation method, we have analyzed two DDEs with rational nonlinearity for solitary and periodic wave solutions. Our approach led to rational solutions for both equations. We verified our results by simulation as well.

\section{References}

[1] E. Fermi, J. Pasta, and S. Ulam, Collected papers of Enrico Fermi, University of Chicago Press, Chicago, 1965.

[2] M. Toda, Theory of nonlinear lattices, Springer-Verlag, New-York, 1989.

[3] K. Narita, $\mathrm{N}$-soliton solution of a lattice equation related to the discrete MKdV equation, J. Math. Anal. Appl. 381 (2011), 963-965.

[4] İ. Aslan, Exact solutions of a fractional-type differential-difference equation related to the discrete MKdV equation, Commun. Theor. Phys. 61 (2014), 595-599.

[5] K. Narita, Solutions for the Mikhailov-Shabat-Yamilov difference-differential equations and generalized solutions for the Volterra and the Toda lattice equations, Progr. Theoret. Phys. 99 (1998), 337-348.

[6] K. Narita, Special solutions to nonlinear difference-differential equations, J. Math. Anal. Appl. 205 (1997), 273-279.

[7] J. S. Russell, "Report on waves," Tech. Rep., 1844, in Proceedings of the14th Meeting of the British Association for the Advancement of Science.

[8] Y. V. Kartashov, V. A. Vysloukh, A. Malomed Boris, and L. Torner, Solitons in nonlinear lattices, Rev Mod Phys. 83 (2011), 247-305.

[9] D. Baldwin, U. Goktas, and W. Hereman, Symbolic computation of hyperbolic tangent solutions for nonlinear differential-difference equations, Comput. Phys. Commun. 162 (2004), 203-217. 